\title{
ACCESO A LOS DATOS PÚBLICOS Y SU REUTILIZACIÓN: OPEN DATA Y OPEN GOVERNMENT
}

\author{
Antonia Ferrer-Sapena, Fernanda Peset y Rafael Aleixandre-Benavent
}

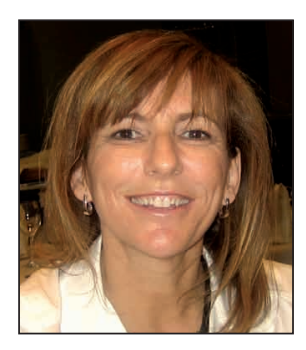

Antonia Ferrer-Sapena es licenciada en geografía e historia contemporánea por la Universidad de Valencia, y doctora en técnicas y métodos de información y documentación. Es coordinadora de investigación en Florida Centre de Formació y miembro de su Comité de Innovación. Entre los proyectos que dirige destaca el portal de economía social EcSocial.com. Es profesora de la Universidad Politécnica de Valencia, y miembro del foro Innovem Junts 2008, del Instituto de la Pequeña y Mediana Industria (Impiva) de la Generalitat Valenciana. Es miembro del Grupo ThinkEPI sobre estrategia y prospectiva de la información.

Univ. Politécnica de Valencia, Depto. de Comunicación Audiovisual, Documentación e Historia del Arte Camino de Vera, s/n. 46022 Valencia http://www.ecsocial.com anfersa@upv.es

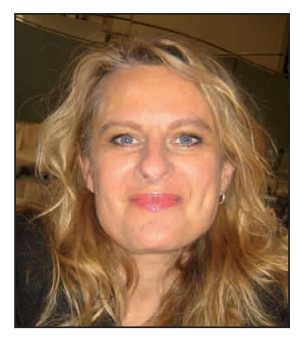

Fernanda Peset es licenciada en geografía e historia y doctora en documentación. Profesionalmente viene del mundo de las unidades de información y desde 1997 es profesora titular de la Universidad Politécnica de Valencia. Su trabajo y publicaciones se orientan a la comunicación científica, acceso abierto e implantación del protocolo OAI-PMH, normalización de la información, descripción de documentos, sistemas de documentación de museos, etc. Es responsable del Posgrado Oficial Industrias Culturales y de la Comunicación. Participa en los proyectos IraLIS, E-LIS, Grupo ThinkEPI y Ciepi.

Univ. Politécnica de Valencia, Depto. de Comunicación Audiovisual, Documentación e Historia del Arte Camino de Vera, s/n. 46022 Valencia mpesetm@upv.es

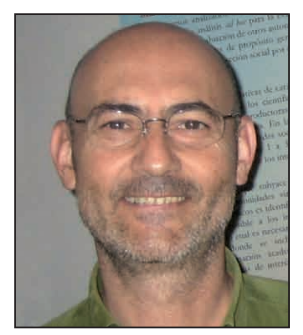

Rafael Aleixandre-Benavent es científico titular del Consejo Superior de Investigaciones Científicas (CSIC), doctor en medicina y especialista en documentación médica por la Universidad de Valencia. Sus principales líneas de trabajo son la evaluación de la investigación y de las publicaciones científicas, los estudios sociales sobre inmigración y la investigación en drogodependencias. Colabora en diversas actividades docentes e investigadoras de las universidades de Valencia, Politécnica de Valencia, Jaume I de Castelló, Universitat de Barcelona y UCAM de Murcia.

Instituto de Historia de la Ciencia y Documentación López Piñero Palacio Cerveró. Plaza Cisneros, 4. 46003 Valencia rafael.aleixandre@uv.es

\section{Resumen}

El open government o gobierno abierto es una forma de comunicación abierta, permanente y bidireccional entre la administración y los ciudadanos, basada en la transparencia por parte de la administración, la colaboración con la sociedad civil y las empresas y en la participación. Se expone uno de los puntos clave que se están utilizando para alcanzar esta participación: el movimiento open data o datos abiertos. En Europa sus orígenes se sitúan en la Directiva 2003/98/CE del Parlamento y del Consejo Europeos sobre el acceso y la reutilización de la información del sector público. Se describen proyectos open data que facilitan el acceso a los datos de la administración de acuerdo con las recomendaciones del W3C. La estructura y formatos abiertos permiten que los datos puedan reutilizarse proporcionando nuevos servicios a ciudadanos y empresas. Se han llevado a cabo numerosos proyectos y aplicaciones de visualización que ya dan cuenta del potencial de este movimiento. La situación en España es incipiente aunque en un corto período de tiempo están apareciendo numerosas iniciativas. Hemos de preguntarnos cuál va a ser el papel que los gestores de información adoptarán en este nuevo escenario.

\section{Palabras clave}

Gobierno abierto, Acceso a la información gubernamental, Acceso a la información de la administración, Datos abiertos, Open data, Open government, Recomendaciones.

\section{Title: Access to and reuse of public data: open data and open government}




\begin{abstract}
Open government is a form of permanent open communication between the administration and citizens, based on transparency from the administration, working with civil society and business. We present one of the key points that are being used to achieve this objective of participation: the open data movement. In Europe, its origins lie in the issuance of Directive 2003/98/EC of the European Parliament and the Council on access to and reuse of public sector information. Open data projects described here provide access to government data according to the recommendations of the $W 3 C$. The structure and open format allows data to be reused to provide new services to citizens and businesses. Numerous projects and visualization applications have been developed that realize the potential of this movement. The situation in Spain is emerging and even in a short period of time many initiatives are maturing. This offers us the opportunity to ask what role information managers will take on in this new scenario.
\end{abstract}

\title{
Keywords
}

Open government, Open data, Open data projects, Access to government information, Recommendations.

Ferrer-Sapena, Antonia; Peset, Fernanda; Aleixandre-Benavent, Rafael. “Acceso a los datos públicos y su reutilización: open data y open government". El profesional de la información, 2011, mayo-junio, v. 20, n. 3, pp. $260-269$.

\section{Introducción. El open government}

Se puede entender el open government o gobierno abierto como una forma de comunicación abierta, permanente y bidireccional entre la administración y los ciudadanos, que pretende conseguir una colaboración real entre todos. A la administración abierta contribuye de manera efectiva la web social o web 2.0, al ofrecer plataformas multidireccionales de comunicación. El gobierno abierto supone una simetría en el diálogo ciudadanos-administración y sus conceptos clave, tal y como señaló Barack Obama en su discurso del 21 de enero de 2009 son transparencia, colaboración y participación (Obama, 2009):

- La transparencia promueve la rendición de cuentas por parte de la administración ante los ciudadanos informando sobre aquello que está realizando y sus planes de actuación.

- Colaboración con los ciudadanos, las empresas y la sociedad civil, permitiendo un trabajo conjunto de la administración con sus trabajadores y la colaboración entre las administraciones.

- Participación, donde la administración, que tiene acceso a conocimiento disperso, debe favorecer su acceso y permitir que los ciudadanos participen de manera activa en la formulación de políticas de forma que su experiencia colectiva y la información beneficie al gobierno. Para ello, la administración debe fomentar y mejorar las oportunidades de participación de los ciudadanos.

\section{El open government o gobierno abierto es una forma de comunicación abierta, permanente y bidireccional entre la ad- ministración y los ciudadanos}

La tecnología influye en este proceso de gobierno abierto, si bien para que se produzca es necesario un cambio cultural y de formación de todos los que intervienen en el proceso: administración y ciudadanos. Estos últimos ya están pre- parados para estos nuevos escenarios. Como muestra, un ejemplo antes inconcebible: en España, la plataforma Access Info exigió una ley de transparencia utilizando la web 2.0 y consiguió que el ministro recibiera a sus representantes en 15 días.

La guía Open government. 10 ideas para hacer tu ayuntamiento abierto (ORSI, 2010), indica las claves a seguir para conseguir un gobierno local más participativo y abierto. Una de las iniciativas que propone es facilitar un acceso a los datos por las siguientes razones:

- La apertura de datos favorece la transparencia y rendición de cuentas.

- Proporciona nuevas oportunidades de negocio para las empresas que crean aplicaciones y utilizan los datos brutos que ofrece la administración.

- Los ciudadanos obtienen mejores servicios basados en los datos ofrecidos por la administración. Se mejora la eficiencia ya que no es necesario gastar recursos en informes y estudios elaborados por la administración.

El acceso a los datos permitirá una administración más transparente, mejores servicios e investigar a menor coste

Más allá de estos planteamientos filosóficos, en estas páginas se pretende ofrecer un análisis en profundidad de los aspectos relacionados con la puesta a disposición de la información. Existen dos puntos de interés para nuestra profesión en un gobierno abierto, pues están relacionados directamente con el acceso a los datos de la administración. Uno de ellos recomienda la apertura de datos públicos, y el otro promueve la elaboración de aplicaciones basadas en datos públicos por parte de terceros. Además, será el acceso a los datos lo que permitirá una administración más transparente, la promoción de mejores servicios y la posibilidad de realizar investigaciones con un menor coste. 


\section{Concepto y orígenes de open data}

La iniciativa open data se enmarca en el contexto de gobierno abierto que hemos mencionado y su filosofía es la del acceso abierto a determinados datos sin restricciones de copyright. En este movimiento se parte de la base de que la información de la Administración es patrimonio de todos los ciudadanos, por lo que les debe ser devuelta. La Administración no siempre tiene capacidad para tratarla, y es aquí donde las empresas pueden intervenir creando servicios de valor añadido que hagan la información más comprensible y asequible.

Una definición reciente describe el open data como "un movimiento que promueve la liberación de datos, generalmente no textuales y en formatos reutilizables como csv (comma separated values), procedentes de organizaciones diversas" (Peset; Ferrer-Sapena; Subirats-Coll, 2011). Según la Open Knowledge Foundation se considera obra abierta si cumple tres condiciones: disponibilidad íntegra de acceso, posibilidad de redistribución, y licencia para la reutilización. En la definición de este término se incluye:

- contenidos como música, películas y libros;

- datos científicos, históricos, geográficos o de cualquier otro tipo;

- información gubernamental y de otras administraciones públicas.

La fundación CTIC (Centro Tecnológico de la Información y Comunicación), en una definición más concreta, considera como open data los datos públicos expuestos en un formato abierto y estándar, de manera que puedan ser empleados para alimentar servicios y aplicaciones útiles para los usuarios, y que se puedan consumir desde cualquier dispositivo fijo o móvil (Valle-Gijón, 2010).

En relación con sus orígenes, la mayoría de iniciativas sobre open data surgieron en el oeste de Europa y en América del Norte, siendo por el momento prácticamente inexistentes en el resto de países, especialmente aquellos que se encuentran en vías de desarrollo. Las primeras experiencias provienen de los gobiernos de los Estados Unidos, Reino Unido y Nueva Zelanda.

En España, los preliminares normativos se encuentran en la Ley $37 / 2007$ sobre Reutilización de la información del sector público, que transpone la Directiva 2003/98/CE del Parlamento y del Consejo Europeos. Esta Ley -todavía poco conocida por existir poca conciencia del potencial del uso de la información del sector público (Grupo Publidoc, 2010)-, regula y fomenta la utilización de los datos generados y custodiados por la administración pública. La Ley indica que "la reutilización de la información del sector público o privado consiste en el uso por parte de las personas físicas (ciudadanos particulares) o jurídicas (empresas) de información generada por organismos del sector público, con fines comerciales o no".

La Directiva del Parlamento Europeo ya incidía en el papel fundamental de los contenidos digitales para promover la sociedad de la información. Establece un marco europeo de reutilización de la información del sector público para garantizar un mercado interior de información basado en con- diciones de transparencia, equidad, proporcionalidad y no discriminación.

El sector público recoge, produce, reproduce y difunde una amplia gama de información de distintos espacios: información social, económica, geográfica, meteorológica, turística, relacionada con la educación y la salud, etc. Dado que en cada país esta información tiene un trato distinto, la Directiva ha tratado de armonizar las normas y las prácticas nacionales de reutilización de los datos. Un aspecto que recoge y que es cuestionado por algunos, es que se pueden establecer tarifas para el acceso a los datos, siempre que no se superen los costes totales de recogida, producción, reproducción y difusión de los documentos y que la obtención de beneficios sea razonable teniendo en cuenta las exigencias de autofinanciación del organismo público de que se trate.

Debe mencionarse también el papel jugado por la Organización para la Cooperación y el Desarrollo Económico $(O C D E)$ : en la Conferencia ministerial sobre el futuro de la economía de internet de esta organización, celebrada en Seúl en junio de 2008, se aprobó la Declaración para el futuro de la economía de internet, que manifiesta el deseo de promover esta economía con apoyo de la innovación del sector privado y de la sociedad civil. Para ello, se aprobó una Recomendación que señala distintas actuaciones de los estados miembros. Una de ellas promueve fomentar la disponibilidad de la información del sector público y su reutilización de manera no discriminatoria; y otra anima la puesta a disposición de los datos mediante la edición de guías de actuación y con mecanismos de protección a la propiedad intelectual. Un tema clave es garantizar que los precios sean transparentes y competitivos, fomentando además la cooperación entre distintos organismos para una mejor recopilación y conservación de los datos y su distribución. También alude a la difusión de las mejores prácticas en materia de reutilización, aspecto que, como veremos más adelante, ya está presente en numerosos proyectos de open data.

Los proyectos open data pueden contener distintos tipos de información y de servicios, pero sólo se consideran como tales los que distribuyen un catálogo de datos de acceso público de acuerdo con las recomendaciones establecidas por el $W 3 C$. Se excluyen de esta denominación los que ofrezcan acceso a los datos pero no sigan esas recomendaciones. Entre los proyectos open data aparecen diferentes momentos de desarrollo. Si observamos los que hemos recogido como ejemplo se puede apreciar que algunos de ellos ofrecen únicamente datos, mientras que otros, más avanzados, proporcionan además servicios de utilidad para los ciudadanos y las empresas.

Aunque las recomendaciones y la legislación tienen ya un cierto tiempo, la puesta a disposición de los datos no se ha producido de una manera rápida. A nivel internacional, una de las medidas más conocidas es la política de la administración estadounidense con el acceso de Obama al gobierno en 2009. En España ha sido el Plan Avanza 2 (2009-2012) el que ha fomentado este acceso a los datos mediante el Proyecto Aporta. 


\section{Estructura de los datos y formatos}

Para que los datos puedan ser útiles para crear nuevos servicios, investigaciones, etc., es necesario que estén dispuestos de una manera que facilite su integración. En este contexto, la mayoría de proyectos open data en curso especifican de qué manera se deben aportar los datos. Todos ellos se basan en las recomendaciones establecidas por el W3C versión 9 de septiembre del 2009, en las que se describen los pasos a seguir para su publicación (W3C, 2009):

1. Los datos deben publicarse en bruto, manteniendo una estructura que permita su uso automatizado (las más conocidas son $\mathrm{xml}$, rdf y csv). Advierte que se eviten los formatos de visualización, ya que éstos serán creados por las aplicaciones de reutilización.

2. Crear un catálogo en línea para que los usuarios puedan conocer qué se ha publicado. Recomienda incluir metainformación como, por ejemplo, la fecha de actualización del fichero, la periodicidad con que se actualiza y el organismo encargado de ello, datos necesarios para contrastar la calidad de la información aportada. Los datos que se ofrezcan deben ser fiables y encontrarse estructurados y documentados, pues en caso contrario tendrían escasa utilidad.

3. Enriquecer los datos en formato xhtml (extensible hypertext markup language). Como es sabido, este lenguaje de marcado está pensado para sustituir el html y su objetivo es avanzar en el desarrollo de la web semántica, donde la información y la forma de presentarla se encuentran separadas. El uso cada vez más habitual de xml permite compartir los datos de manera más segura y fiable en todas las aplicaciones y soportes, lo que garantiza la compatibilidad. En definitiva, el xhtml combina la sintaxis del html, diseñado para mostrar los datos, con la del $\mathrm{xml}$, diseñado para describirlos.

http://www.desarrolloweb.com/articulos/449.php http://www.w3c.es/divulgacion/guiasbreves/XHTML

Para que los datos puedan ser útiles es necesario que estén dispuestos de una manera que facilite su integración

Estas recomendaciones permiten que los datos puedan convertirse posteriormente en formato linked data (datos enlazados). Un ejemplo de proyecto en el que se realiza una exposición detallada de todos los formatos en los que se pueden aportar los datos es Datos abiertos Gencat. En su web se enumeran todos ellos, diferenciando los de tipo propietario frente a los libres:

- Propietarios: doc (Word), xls (Excel).

- Libres: pdf (formato de documento portable), odf (formato de documento abierto para aplicaciones ofimáticas Oasis), csv (valores separados por coma), xml (lenguaje de etiquetado extensible), tmx (translation memory exchange), json (notación de objetos de javascript), rdf-xml (infraestructura para descripción de recursos), kml-kmz (lenguaje de etiquetado de ojo de cerradura).
Por otro lado, existen múltiples lenguajes para consultar los archivos de datos. El más estandarizado es sparql (protocolo simple y lenguaje de consulta de rdf), promovido por el propio $W 3 C$. Se trata de un lenguaje de consulta para rdf que se está extendiendo como estándar y que permite obtener muy buenos resultados en búsquedas realizadas en una extensa y variada información: datos personales, técnicos, empresariales, científicos, redes sociales...

\section{Acceso a los datos y su reutilización}

Toda esta política de puesta a disposición de la información no tiene sentido si posteriormente la información no es tratada y gestionada para crear estudios, servicios o generar conocimiento en nuestra sociedad. Hay que distinguir dos aspectos fundamentales: por una parte, el acceso a los datos y a qué tipo de datos se puede acceder y, por otra, las posibilidades de su reutilización.

Las posibilidades de acceso a la información generada por los gobiernos pueden ser múltiples, pero, como es lógico, este acceso se encuentra limitado en el caso de acceder a información personal confidencial, secretos de gobierno, secretos militares, patentes en trámite de examen, etc., es decir, información cuyo acceso se encuentra protegido por las leyes.

La puesta a disposición de los datos no tiene sentido si posteriormente no se tratan para generar conocimiento

Existen distintas posturas en cuanto a los límites que deben establecerse en el acceso a la información. Hay quienes reivindican el acceso total a las bases de datos generadas por la administración, ya que las consideran propiedad de los ciudadanos, mientras que otros estiman suficiente el acceso a los datos servidos por la administración pública, aunque no se permita el acceso total a sus bases de datos.

EI W3C también ofrece recomendaciones sobre las políticas de acceso. En ellas se trata qué tipo de información se debe hacer pública, la necesidad de publicar los usos previstos de la misma, los créditos, las limitaciones y las responsabilidades que se tienen al utilizarla y recomienda emplear las licencias Creative Commons http://www.w3.org/TR/gov-data http://es.creativecommons.org

La mayoría de las administraciones expone cuáles son sus políticas de reutilización, dada la multitud de aplicaciones y servicios que se pueden generar. En Reino Unido, por ejemplo, indican en qué casos pueden o no utilizarse. Como es lógico, prohíbe su uso para actividades ilegales, violentas, racistas, cambiar o tergiversar los datos, etc. Se puede publicar, distribuir, adaptar la información y explotarla comercialmente si se combina con el uso de otros datos. Las empresas deben citar la fuente de los datos y, a ser posible, hacer un link a la licencia de uso.

En España, la Ley 37/2007 de Reutilización de información pública hace referencia a la posibilidad de establecer dife- 
rentes licencias para esta información. Considera que la información es un recurso esencial para el desarrollo social y económico (Comisión Europea, 1998) y su utilización puede generar valor y riqueza. Al igual que la Directiva europea, trata la posibilidad de establecer tasas por el uso de los datos y permite sancionar a aquellos que incumplan las licencias de uso. Establece que: "La información generada desde las instancias públicas, con la potencialidad que le otorga el desarrollo de la sociedad de la información, posee un gran interés para las empresas a la hora de operar en sus ámbitos de actuación, contribuir al crecimiento económico y la creación de empleo, y para los ciudadanos como elemento de transparencia y guía para la participación democrática".

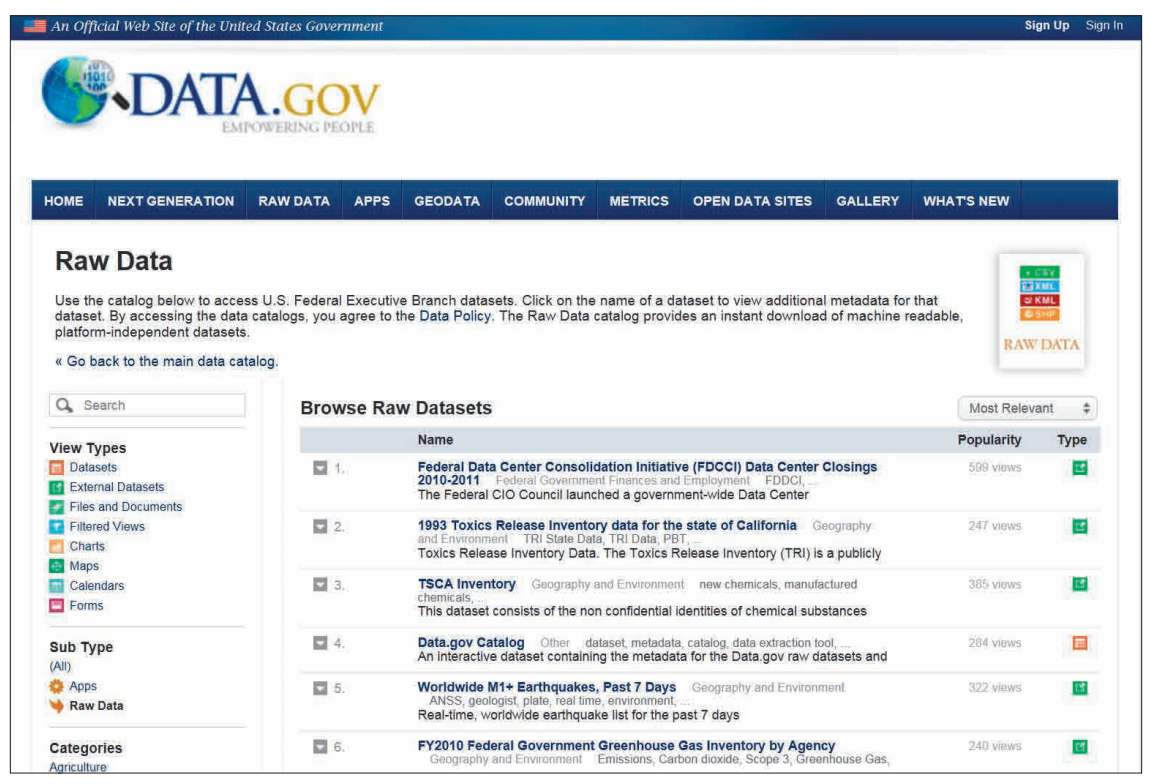

Figura 1. Catálogo de datos del Gobierno de los EUA, http://www.data.gov/catalog/raw

Los beneficios que aporta la reutilización de la información en el sector público a ciudadanos, empresas y administración pública han sido descritos en la Guía Aporta (Secretaría de Estado de Telecomunicaciones, 2009). Ofrece a los ciudadanos la posibilidad de mejorar el conocimiento de las instituciones públicas, haciéndolas más transparentes, cercanas y participativas. En esta libre disposición de los datos, la Ley $37 / 2007$ no se refiere únicamente a los procedimientos políticos, sino también a los judiciales, económicos y administrativos.

Tanto la Directiva 2003/98/CE del Parlamento Europeo y del Consejo, como la Ley 37/2007, marcan la importancia de la reutilización por parte de las empresas como elemento fundamental para incentivar la economía, poniendo en valor los datos de la administración y generando nuevos productos o servicios en un entorno digital. Otro de los intereses de la Ley española, al igual que hacía la Directiva a escala europea, es armonizar la explotación de la información de la Administración. Se dirige especialmente a la información recopilada en formato digital por sus distintos organismos y relativa a distintos ámbitos, con el objeto de reforzar un uso transfronterizo de los documentos por parte de los ciudadanos y las empresas.

\section{Ejemplos de datos abiertos}

Existe el registro $C K A N$ de open data de carácter general mantenido por la OKF (Open Knowledge Foundation), que contiene 1.705 datasets registrados, agrupados temáticamente. Los que más grupos de datos ofrecen se encuentran en la categoría Linked open data, con 85 conjuntos, Datos bibliográficos con 42, y la categoría Where does my money go? con 34.

http://ckan.net

Los proyectos open data que se exponen a continuación son ejemplos representativos a nivel internacional que se pueden agrupar en las siguientes categorías: proyectos de gobiernos nacionales, proyectos de gobiernos municipales y ejemplos de reutilización de datos por parte de empresas

e instituciones de carácter privado. Por último, se presentan algunos ejemplos realizados en España.

\subsection{Proyectos de gobiernos nacionales}

Uno de los ejemplos a destacar entre los proyectos de apertura de datos es el modelo de la Administración de los Estados Unidos de Barack Obama, que en su agenda TIC internacional señaló que "Un solo bit de información puede generar un torrente de creatividad" (Solana, 2011). No hay que olvidar el origen de EUA, que nació con la noción de soberanía popular, por lo que tienen claro que los datos del gobierno son datos de los ciudadanos. El acceso a la información pública es un derecho, y cualquier documento creado por el gobierno debe ser de dominio público sin restricciones de derechos de autor, aspecto que se contrapone a la mentalidad y regulación de los estados europeos donde es el gobierno el que decide qué datos y en qué forma se pueden publicar (Eaves, 2009). El Gobierno americano dispone, después de más de un año de funcionamiento, de más de 380.000 datasets, ejemplo que se está imitando en otros países y ciudades. http://data.gov

Algunos ejemplos de aplicaciones que se han desarrollado con los datos públicos son

- FlyOnTime: ofrece información sobre vuelos en tiempo real;

- National obesity comparison tool: ofrece mapas comparativos de obesidad entre los distintos estados americanos;

- Employment market explorer: útil para entender el mercado local de empleo regional y estatal, permitiendo analizar la dinámica del mercado laboral;

- Clean air status and trends network: estado de limpieza del aire en distintos lugares del país.

Estos datos abiertos, además de ofrecer nuevos servicios, sirven para que la Administración sea mucho más transparente para los ciudadanos. Así, algunas plataformas cívicas han creado aplicaciones de interés como la Transparency data, creada por la Sunlight Foundation de los EUA, que permite localizar las contribuciones económicas recibidas por los distintos candidatos políticos estadounidenses. Los datos se encuentran referidos a campañas federales y esta- 
tales celebradas en el país desde 1989 hasta 2010. Otra aplicación permite la visualización del censo de los EUA. http://transparencydata.com

Otro ejemplo es el del gobierno de Reino Unido, mucho más ágil que el estadounidense a la hora de poner los datasets a disposición de los ciudadanos. Actualmente dispone de más de 6.900 que ofrecen información sobre la política del gobierno, permitiendo que se conozcan en detalle sus actuaciones y que los ciudadanos entiendan cómo funcionan y cómo se formulan sus políticas. La información ofrecida nutre algunas webs de gran popularidad, como Where does my money go? que muestra dónde va el dinero público. En España ha sido imitada por la página ¿Dónde van mis impuestos? Aunque con la misma filosofía, la información que ofrecen no es exactamente igual, pero sí que permite visualizar los gastos anuales de la Administración Central del Estado y la Seguridad Social, tal y como se recoge en los Presupuestos Generales del Estado.

http://data.gov.uk

http://wheredoesmymoneygo.org http://dondevanmisimpuestos.es

El gobierno de Reino Unido ha sido un gran defensor y promotor de la innovación en la incorporación de datos al público y ha propiciado el concurso Show it a better way, donde se premian las mejores iniciativas para mashups de acceso a datos gubernamentales. En España se realiza un concurso similar de 48 horas llamado Abredatos para el desarrollo exprés de servicios tecnológicos al ciudadano. http://data.gov.uk

\subsection{Proyectos de gobiernos municipales}

Los municipios han puesto en marcha numerosas iniciativas en este campo, lo cual no es de extrañar ya que son las administraciones más cercanas al ciudadano y las que pueden ofrecerle una información más directa y relacionada con su día a día.

Algunos proyectos de administraciones municipales son los siguientes:

Ciudad de Vancouver. Dispone de un catálogo importante de datos públicos en una tabla ordenada alfabéticamente donde aparece el tipo o tipos de ficheros disponibles. Se presentan webs relacionadas con los datos ofrecidos y se puede conseguir información sobre, por ejemplo, rutas de bicicletas donde el tráfico de coches es menor, el aire es más limpio, seguras, etc., etiquetadas con signos. http://data.vancouver.ca/datacatalogue/index.htm

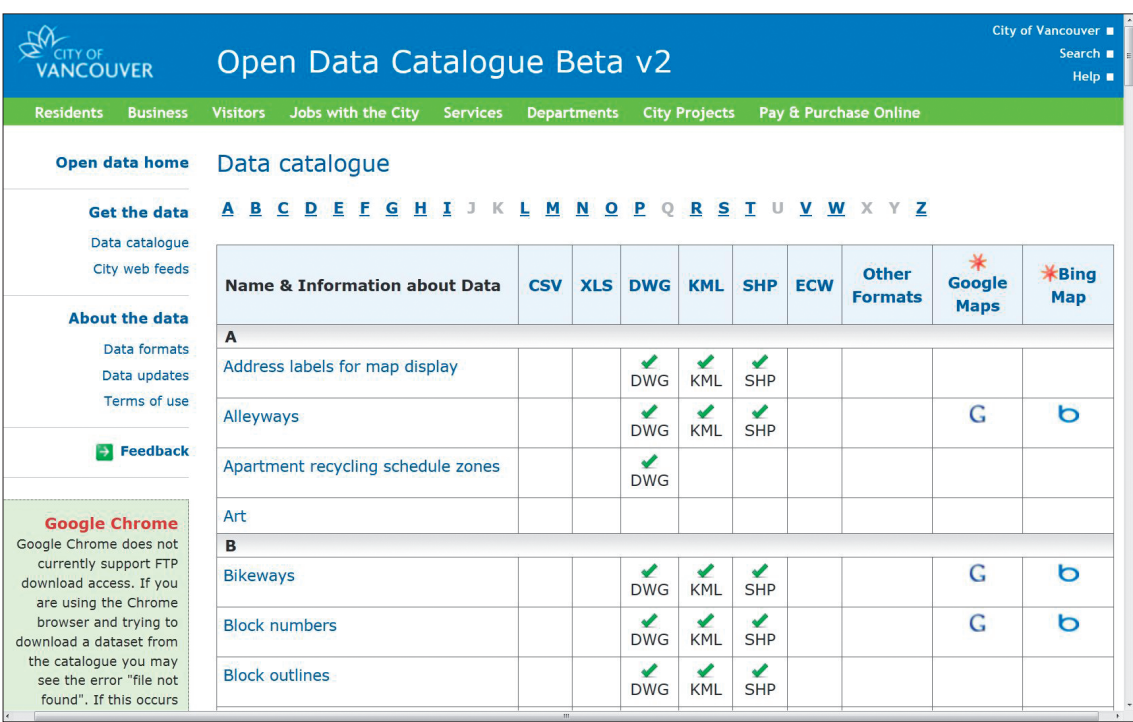

Figura 2. Catálogo de datos de Vancouver, http://data.vancouver.ca/datacatalogue/index.htm

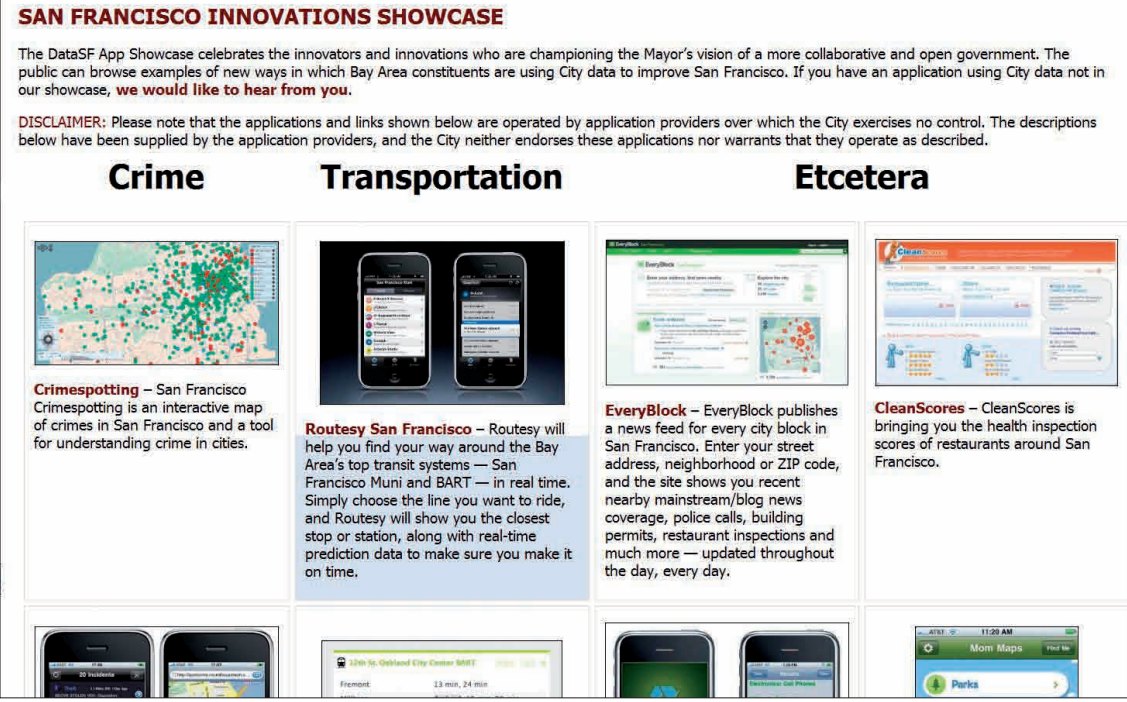

Figura 3. Aplicaciones de los datasets de San Francisco, http://datasf.org/showcase

Ciudad de San Francisco. Ofrece datasets muy variados, como la localización de las redes inalámbricas, los enclaves de la ciudad que han aparecido en películas desde 1924 a 2010 (ofreciendo datos de título de la película, actores que aparecen en las escenas, etc.).

http://www.datasf.org

Su web contiene aplicaciones como:

- Crimespotting, que informa de las partes de la ciudad donde existe mayor inseguridad;

- Routesy San Francisco, aplicación que permite conocer en tiempo real dónde se encuentra el medio de transporte más adecuado para llegar al lugar que se quiere, con una aproximación del tiempo que se tardará en llegar según el tipo de transporte que se utilice;

- EveryBlock, noticias sobre cada bloque o manzana de la ciudad. Al introducir el código postal informa de las últi- 
mas noticias, las llamadas realizadas a la policía, los permisos de construcción, inspecciones a restaurantes, etc. (EveryBlock funciona también en Chicago, New York, Philadelphia, Dallas, Washington, Boston y Seattle).

- The original parking locator, aplicación que permite localizar el coche en el parking donde se haya aparcado, indicando lugar, planta, camino más corto para llegar, etc.

Ciudad de Washington DC. Proporciona acceso a 472 bases de datos de distintas agencias y permite la suscripción a varios canales rss, ofreciendo los datos en $\mathrm{xml}$, texto, csv, kml o en formato Esri-Shapefile. Algunos de sus datos son visualizados desde la web ManyEyes de IBM. Presenta visualizaciones sobre las 100 principales empresas de tecnología en la ciudad, dónde se encuentran las tiendas de comestibles y de salud, la distribución del presupuesto de la ciudad para salud y personas vulnerables, entre otras.

http://data.octo.dc.gov

Ciudad de Nueva York. La información sobre los 217 datasets liberados (abril 2011) se puede localizar mediante un buscador. Se encuentran organizados por categoría y agencias de la ciudad. http://www.nyc.gov/html/datamine/ html/data/raw.shtml

\subsection{Aplicaciones de visualización de datos}

Algunos proyectos que recogen $d a-$ tasets y brindan visualizaciones son (MacDonald; Martínez, 2008):

- Data360: web colaborativa de código abierto en la que se recogen datos de todo el mundo. Dispone de una base de datos común y compartida que permite que cualquier persona u organización comprometida con la neutralidad y el no-partidismo pueda utilizar la presentación de informes y la visualización de los datos. http://www.data360.org

- ManyEyes: utilidad de IBM que pretende democratizar la visualización de datos y lograr que pueda acceder al análisis de los mismos una nueva clase social.

http://www-958.ibm.com/software/data/cognos/manyeyes

- Gapminder: fundación sueca que en colaboración con el Departamento de Estadística de las Naciones Unidas ha elaborado un software que permite la visualización de datos estadísticos con 16 variables. El software ha sido adquirido por Google para la visualización de datos y animaciones que aparecen en su Google Public Data. http://www.gapminder.org/desktop

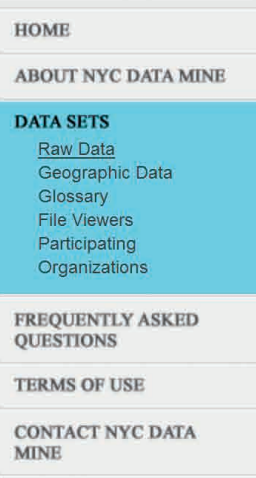

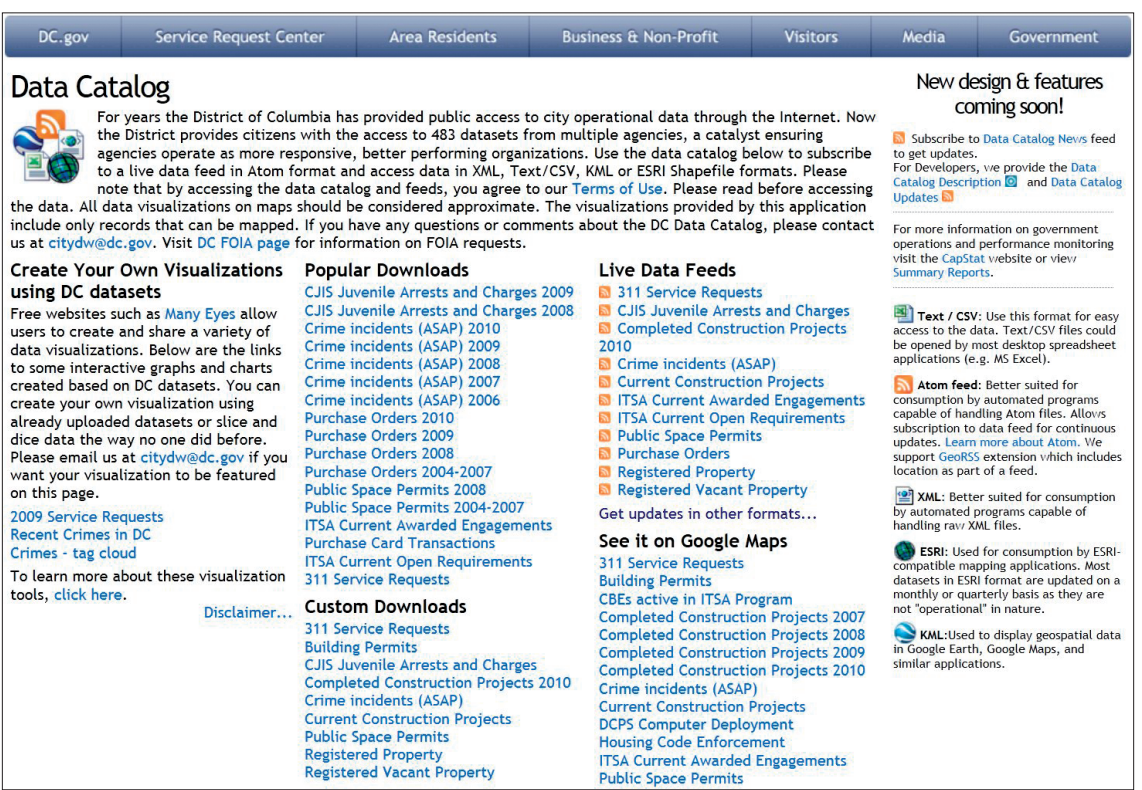

Figura 4. Datasets de Washington, http://data.octo.dc.gov

Search | Email Updates | Contact Us

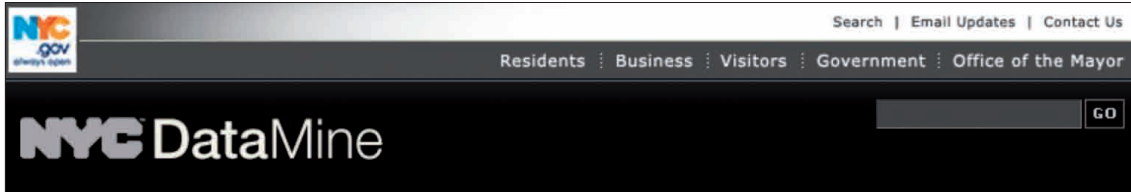

自 Translate this Page | $\triangle$ Email a Friend | Text Size: A A A

Raw Data

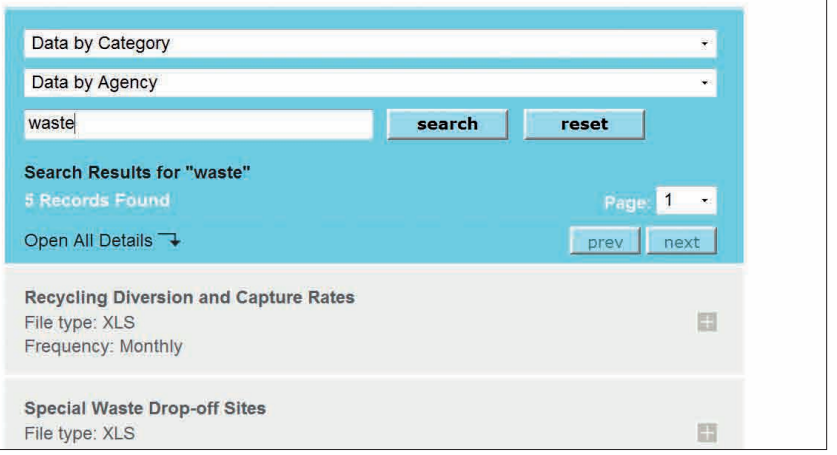

Figura 5. Pantalla de búsqueda de datasets del Ayuntamiento de Nueva York, http://www.nyc.gov/ html/datamine/html/data/raw.shtml

- Geocommons: para visualizar datos geográficos en mapas elaborados con mashups y crear mapas temáticos. Se generan con más de 50.000 datos en código abierto dispuestos en Geocommons y pueden ser fácilmente embebidos en aplicaciones de Facebook, Twitter, etc.

http://geocommons.com

- Socrata: ejemplo comercial de reutilización de datos donde, previo pago, se pueden cargar y visualizar datos públicos conjuntos, acceder a los mismos mediante una API y difundirlos en redes sociales. http://www.socrata.com

\subsection{Situación en España}

Para apoyar la Ley 37/2007 sobre Reutilización de la información del sector público nació el Proyecto Aporta. Se enmarca dentro de Plan Avanza 2, que tiene como objetivo la eliminación del papel en la Administración Pública para el período 2010-2015. El primer Plan Avanza tenía como 
objetivo fundamental incrementar la cobertura y conectividad de la Red en España, intentando con ello recuperar el retraso existente en relación con el resto de Europa. Este segundo Plan pretende conseguir productos y servicios TIC avanzados, donde uno de los puntos fuertes es el proyecto de eAdministración (Marcos-Martín; SorianoMaldonado, 2011). http://www.aporta.es

En España, la Fundación CTIC, Gijón, colabora y lleva a cabo proyectos open data. La mayoría de sus iniciativas han dado como resultado portales públicos en los que se ofrece información de la administración. Ejemplos de ello son los realizados en el Gobierno Vasco con el proyecto Irekia, el proyecto del Gobierno del Principado de Asturias (Datos Asturias), el de la Generalitat de Catalunya (Dades obertes), o la del Ayuntamiento de Zaragoza (Datos de Zaragoza). Las realizaciones existentes pueden observarse en la figura 6. El catálogo de datos abiertos de España se puede consultar desde la web del Proyecto Aporta.

http://www.aporta.es/web/guest/buscador_de_catalogos

A partir de esta decisión de apertura de datos de la Administración pública han nacido distintas ideas para la elaboración de iniciativas innovadoras para su reutilización. Como se ha comentado antes, en España Abredatos estimula la creación de aplicaciones de uso de los datos mediante premios a las mejores iniciativas.

Los proyectos pioneros en nuestro país han sido:

\section{- Dades obertes de la Generalitat de Catalunya}

Enfocado hacia las tecnologías linked data, publica conjuntos de datos en rdf. Dispone de datos semánticos de más de 26.000 edificios públicos (vCard en rdf), más de 700 trámites y servicios y otros muchos datos en formatos estructurados y reutilizables. Hay que señalar la representación del catálogo de datos usando el vocabulario internacional Dataset catalog (dcat). Es un vocabulario estándar de descripción de datos que está desarrollando el grupo de trabajo de eGovernment de W3C para permitir la interoperabilidad con otros sistemas.

http://vocab.deri.ie/dcat

- Open data Euskadi del Gobierno del País Vasco

Ofrece un catálogo de datos muy variado que incluye actividades económicas, administración pública, medio ambiente e información estadística.

- Gobierno del Principado de Asturias

Ha sido pionero en elaborar el proyecto íntegramente desde el análisis de datos mediante una herramienta que permite la creación de un almacén semántico. Ha sido el primero a nivel mundial en estar creado íntegramente con tecnología linked data.

- Ayuntamiento de Zaragoza

Fue el primer ayuntamiento en España en abrir sus datos al público. Los datos también se encuentran estructura-

dos y modelados siguiendo linked data y cuenta con un acceso de consulta sparql. Al igual que la Generalitat de Catalunya, utiliza el vocabulario dcat. Entre las aplicaciones desarrolladas con la reutilización de datos se encuentra el planificador de rutas turísticas.

Otras administraciones locales que llevan a cabo proyectos de datos abiertos son los ayuntamientos de Barcelona, Gijón, Lleida y Badalona. A nivel autonómico se están incorporando los gobiernos de las Islas Baleares y el de Extremadura. El Ayuntamiento de Barcelona, tal y como indicaba el periódico Público el 23 de noviembre de 2010 (Criado, 2010), tiene prevista la liberación de toda su información para que pueda ser reutilizada por los ciudadanos.

Todos estos proyectos permiten ver cómo España está entrando en una dinámica activa en la liberación de datos públicos que en algunos casos es semejante o incluso mayor que la de otros países que comenzaron antes este tipo de iniciativas. El localizador de datos abiertos a nivel mundial de la web de la Fundación CTIC ofrece una perspectiva clara de la situación. El mapa incluye 64 datasets públicos, de los cuales 11 son españoles. En Europa está pendiente la elaboración de un catálogo conjunto de datos de los distintos países de la Unión.

http://datos.fundacionctic.org/sandbox/catalog/faceted

La liberalización de los datos va a suponer un cambio organizativo, estructural y de la forma de trabajar de las administraciones

\section{Conclusiones}

El movimiento de apertura de datos de la administración no puede ser tomado a la ligera ya que, como se ha visto, sus beneficios son apreciables: acceso a indicadores de rendimiento de las distintas áreas del gobierno central, autonómico o local, información sobre proveedores de la admi- 


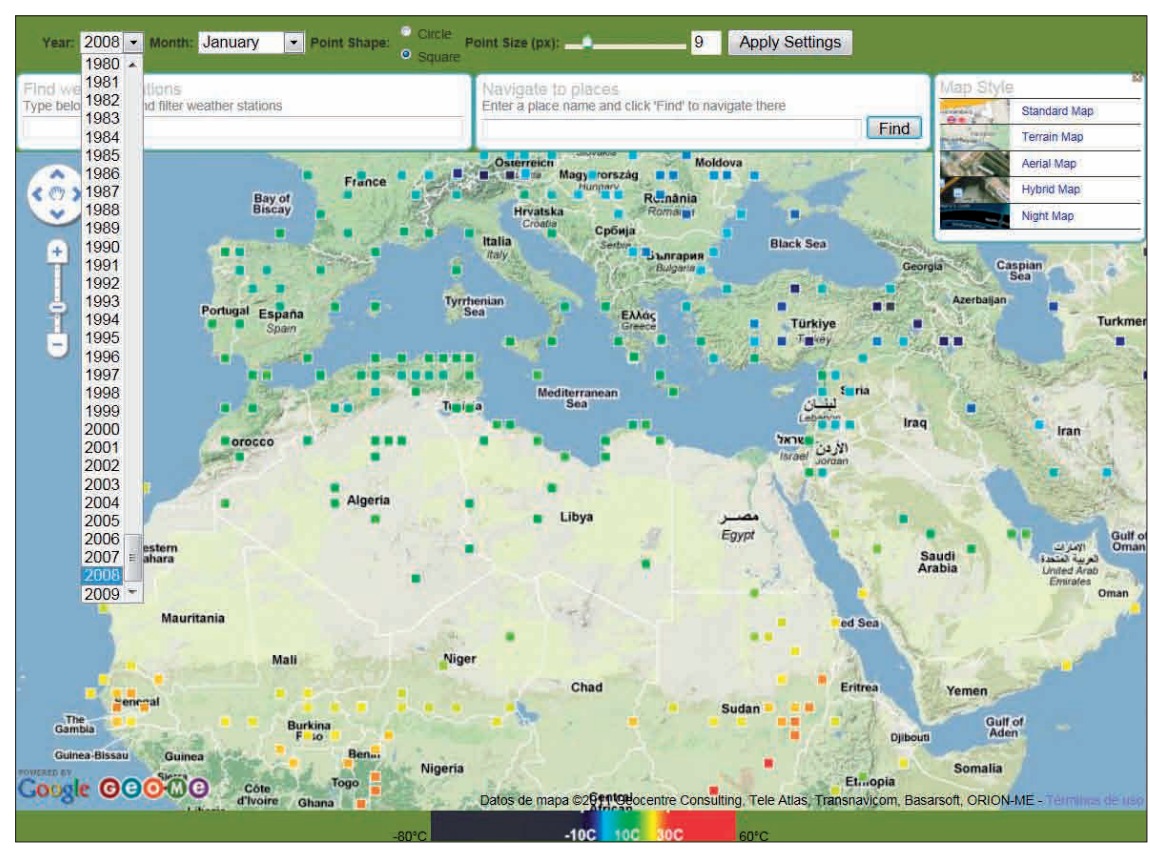

Figura 7. Mashup de Google Maps con datos abiertos suministrados por el servicio de meteorología del Reino Unido (UK Met Office). Los colores de los cuadritos señalan las temperaturas medias mensuales registradas desde el s. XVIII por 1.500 estaciones meteorológicas de todo el mundo. En la imagen se muestra el mes de enero de 2008, http://labs.geo.me/climate_trends

la información de la administración está al servicio de los ciudadanos y se nutre de los impuestos que pagamos.

Para finalizar, hagámonos la siguiente pregunta, ¿deben las bibliotecas adoptar un papel activo en la preservación y puesta en servicio de esta información? Nuestra opinión es que las bibliotecas no pueden permanecer indiferentes al movimiento de datos abiertos. A lo largo de los últimos años se han tratado de manera casi cotidiana los estándares abiertos, el software de código abierto, el acceso libre a las publicaciones y actualmente el acceso libre a los datos. Un ejemplo en el que puede apreciarse su papel activo es el proyecto DISC-UK Datashare (Data Information Specialist Committee) del Reino Unido, cuyo objetivo es que las bibliotecas de investigación del país sirvan como preservadoras de los datos de apoyo a la gestión y a las actividades de investigación mediante la

nistración, sobre las licitaciones concedidas, etc., aspectos que permiten tener un gobierno más abierto y transparente. Además, el acceso a los datos no sólo incluye beneficios a ese nivel, sino que la generación de aplicaciones que los reutilizan aportan nuevos servicios a los ciudadanos: callejeros y rutas personalizadas, localización de empresas, puntos de reciclaje, puntos más conflictivos de una ciudad, o conocer en tiempo real los horarios de los transportes y desviaciones que puedan sufrir del horario previsto, etc.

La liberalización de los datos va a suponer un cambio organizativo, estructural y en la forma de trabajar de las administraciones. Éstas tienen que establecer unas políticas de uso y privacidad sobre los datos que vayan a liberar, a cambio de ahorrar ciertos costes en la elaboración de los informes por parte de terceros.

Las políticas de liberación de datos que se están articulando en España no se están acompañando de un gobierno participativo y no parece ser ese su objetivo por ahora. Ello puede deberse a que los datos liberados por las distintas administraciones se dirigen más a estimular y probar nuevos servicios y utilidades que a fomentar una forma innovadora de gobierno, el gobierno abierto. Hay que tener en cuenta, además, la falta tradicional de una cultura participativa, algo que está cambiando por el uso masivo de las herramientas de la web 2.0.

La liberación de datos supone una oportunidad para que las pymes tecnológicas y creativas puedan crear aplicaciones. España, aunque se encuentra en una fase incipiente, ocupa un puesto avanzado en relación a otros países gracias al número de proyectos que se han puesto en marcha.

Un aspecto relevante, tanto para las empresas que crean aplicaciones como para el ciudadano, es si la administración puede solicitar el pago por el acceso a la información. Esta posibilidad debe cuestionarse y ser objeto de debate, ya que creación de repositorios abiertos institucionales y las tecnologías de la web 2.0. El proyecto nació en 2004, cuando un grupo de bibliotecarios estadísticos trataron de compartir los datos científicos de cuatro instituciones académicas de Edimburgo, Oxford, Southampton y la London School of Economics, comenzando a trabajar con los datos de manera similar a la de los repositorios. En definitiva, en una sociedad en la que los estudios más recientes demuestran que la cantidad de información se va a duplicar durante los próximos 5 años, y donde los peligros de preservación y acceso a la misma juegan un papel fundamental, las bibliotecas deben adaptarse a este nuevo entorno y facilitar el acceso abierto a los datos.

¿Deben las bibliotecas adoptar un papel activo en la preservación y puesta en servicio de este tipo de información?

\section{Referencias}

Access Info.

http://www.access-info.org

CensusMaps 2010.com, Moonshadow Mobile, 2011. http://censusmaps2010.com

CKAN - The Data Hub. http://ckan.net

Comisión Europea. La información del sector público: un recurso clave para Europa. Libro verde sobre la información del sector público en la sociedad de la información. Bruselas, 1998.

http://ec.europa.eu/information_society/policy/psi/docs/ pdfs/green_paper/gp_es.pdf 
Criado, Miguel-Ángel. "Barcelona liberará sus datos públicos". Público, 2010, 23 noviembre.

http://www.publico.es/ciencias/348044/barcelona-libera ra-sus-datos-publicos

Datos abiertos de Zaragoza. http://www.zaragoza.es/ciudad/risp

Datos abiertos Gencat. Proyecto de apertura de datos públicos (open data) de la Generalitat de Catalunya.

http://dadesobertes.gencat.cat/es/formats.html

Datos de Asturias.

http://www.asturias.es/portal/site/Asturias/menuitem.77b $6558 \mathrm{ac} 8616446 \mathrm{e} 445310 \mathrm{bb30a0a0} /$ ?vgnextoid $=05 \mathrm{badd} 4$ 2ece45210VgnVCM10000097030a0aRCRD\&vgnextchannel =05badd42ece45210VgnVCM10000097030aOaRCRD\&i18n. http.lang=es

Dcat vocabulary resources \& examples. http://vocab.deri.ie/dcat-overview

Eaves, David. "Open data - USA vs Canada". Eaves.ca: if writing is a muscle, this is my gym. 8 octubre 2009.

http://eaves.ca/2009/10/08/open-data-us-vs-canada

Grupo Publidoc-UCM. Información del sector público (ISP): Identificación de posibles acuerdos exclusivos - España, Informe final.

http://multidoc.rediris.es/ppo/acuerdos-exclusivos-isp-pu blidoc.pdf

Guía Aporta sobre reutilización de la información en el sector público. Madrid: Secretaría de Estado de Telecomunicaciones y para la Sociedad de la información, 2009.

http://www.aporta.es/web/guest/form_descarga_aporta

Ley 37/2007, de 16 de noviembre, sobre Reutilización de la información del sector público. BOE n. 276, pp. 4716047165.

MacDonald, Stuart; Martínez-Uribe, Luis. "Libraries in the converging worlds of open data, e-research and web 2.0". Online, Mar.-Apr., 2008, n. 32, v. 2, pp. 36-40.

http://ie-repository.jisc.ac.uk/227/1/Online_mar08.pdf

Marcos-Martín, Carlos; Soriano-Maldonado, Salvador-Luis. "Reutilización de la información del sector público y open data en el contexto español y europeo. Proyecto Aporta". El profesional de la información, 2011, mayo-junio, v. 20, n. 3, pp. 291-297.

DOI: 10.3145/epi.2011.may.07

Obama, Barack. Transparency and open government: memorandum for the heads of executive departments and agencies, 2009.

http://www.whitehouse.gov/the_press_office/Transparen cyandOpenGovernment

Observatorio Regional de la Sociedad de la Información de Castilla y León (ORSI). Red de Municipios Digitales de Castilla León. Open government. 10 ideas para hacer tu ayuntamiento abierto. http://issuu.com/orsicyl/docs/open_government._10_ideas para_hacer_tu_ayuntamien?mode $=a \_p$

Opendata@CTIC: sobre la apertura y la reutilización de los datos.

http://datos.fundacionctic.org/

Open government licence for public sector information.

http://www.nationalarchives.gov.uk/doc/open-govern ment-licence/

Open Knowledge Foundation. Definición de Conocimiento Abierto v.1.0.

http://www.opendefinition.org/okd/espanol/

OpenData BCN (apertura de datos públicos en el Ayuntamiento de Barcelona).

http://w3.bcn.es/V01/Serveis/Noticies/V01NoticiesLlistat NoticiesCtl/0,2138,1013028362_1118177754_2_13954460 99\%20,00.html

"Opening up government". HM Government. http://data.gov.uk/codeofconduct

Parlamento Europeo. “Directiva 2003/98/CE del Parlamento Europeo y del Consejo de 17 de noviembre de 2003 relativa a la Reutilización de la información del sector público". Diario Oficial de la Unión Europea, 2003, 31 diciembre, pp. L 345/90-L 345/96.

Peset, Fernanda; Ferrer-Sapena, Antonia; Subirats-Coll, Imma. "Open data y linked open data: su impacto en el área de bibliotecas y documentación". El profesional de la información, 2011, marzo-abril, v. 20, n. 2, pp. 164-172.

Planifica tu visita.

http://www.zaragoza.es/turruta/Turruta/index_Ruta

Solana, Anna. "Liberando datos para construir ciudades más inteligentes". La vanguardia, 2011, 12 febrero.

http://www.lavanguardia.es/internet/20110212/54114005 322/liberando-datos-para-construir-ciudades-mas-inteligen tes.html

Sunlight Foundation. Making government transparent \& accountable.

http://sunlightfoundation.com

Valle-Gijón, Rosa. "EI CTIC convierte a Asturias en sede mundial del open data". Innova especial innovación y nuevas tecnologías. 2010, diciembre.

http://ct.ctic.es/web/contenidos/es/CTICalDia/salaDePren sa/noticias/noticia_0374.html

W3C. "El lenguaje de consulta sparql para RDF es candidato a recomendación". W3C España, 14 junio 2007.

http://www.w3c.es/Noticias/2007/06/14/el-lenguaje-deconsulta-10/

W3C. "Publishing open government data". W3C working draft, 2009.

http://www.w3.org/TR/2009/WD-gov-data-20090908/ 